Bull. Austral. Math. Soc.

42B $20,44 \mathrm{~A} 15,47 \mathrm{~B} 38$

Vol. 46 (1992) [475-478]

\title{
FINITE HILBERT TRANSFORMS AND COMPACTNESS
}

Susumu OKada

It is shown that for the finite Hilbert transform $T_{p}$ on the Banach space $\mathcal{L}^{p}(]-1,1[)$, $1<p<\infty$, the linear operator $T_{p}^{n}+I$ is not strictly singular whenever $n$ is a positive integer.

\section{INTRODUCTION}

Let $1<p<\infty$. The Hilbert transform $H_{p}$ on the space $\mathcal{L}^{p}(\mathbb{R})$ is defined by the Cauchy principal value

$$
\left(H_{p} f\right)(t)=\lim _{\varepsilon \neq 0}\left[\int_{-\infty}^{t-\varepsilon}+\int_{t+\varepsilon}^{\infty}\right] \frac{f(\tau)}{\pi(\tau-t)} d \tau, \quad t \in \mathbb{R}
$$

for every $f \in \mathcal{L}^{p}(\mathbb{R})$. Then $H_{p}$ is a continuous linear operator satisfying the $M$. Riesz identity: $H_{p}^{2}+I=0$ on $\mathcal{L}^{p}(\mathbb{R}),[6$, p.239].

Let $\Omega$ denote the open interval ]-1, 1[. It is clear that the identity $T_{p}^{2}+I=0$ does not hold for the finite Hilbert transform $T_{p}$ on $\mathcal{L}^{p}(\Omega)$ (for the definition of $T_{p}$, see Section 2). For example,

$$
\left(T_{p}^{2}+I\right)\left(\left(1-\mathbf{x}^{2}\right)^{1 / 2}\right)=-\pi^{-1}\left(2+x \ln (1-x)(1+x)^{-1}\right)+\left(1-x^{2}\right)^{1 / 2} \neq 0
$$

$\mathbf{x}$ denoting the identity function on $\Omega$. If one believes that the finite Hilbert transform behaves like the Hilbert transform, then $T_{p}^{2}+I$ ought to be a "small" operator. Therefore it would be natural to see whether or not $T_{p}^{2}+I$ is compact. This question has been raised by $M$. Cowling.

The aim of this note is to show that, given a positive integer $n$, the operator $T_{p}^{n}+I$ on $\mathcal{L}^{p}(\Omega)$ is not strictly singular, and hence it is not compact; see Theorem 3.

Received 28 November 1991

The author thanks Professor David Elliott and Dr. Brian Jefferies for discussions on the theme of this note. He also acknowledges the support of the Australian Reaearch Council and a University of New South Wales Grant.

Copyright Clearance Centre, Inc. Serial-fee code: 0004-9729/92 \$A2.00+0.00. 


\section{ThE MAIN RESULT}

Let $X$ be a Banach space. A continuous linear operator $S: X \rightarrow X$ with closed range is called a Noether or Fredholm operator if the dimension of its null space $\mathcal{N}(S)$ and the codimension of its range $\mathcal{R}(S)$ are both finite. The index $\kappa(S)$ of a Noether operator $S$ is defined as

$$
\kappa(S)=\operatorname{dim} \mathcal{N}(S)-\operatorname{codim} \mathcal{R}(S)
$$

A continuous linear operator $A: X \rightarrow X$ is called strictly singular if the restriction of $A$ to any infinite-dimensional subspace of $X$ is not an isomorphism onto its range. In particular, compact operators are strictly singular.

The following result can be found in [3, Propositions 2.c.7 and 2.c.10], for example.

Lemma 1. Let $S$ be a Noether operator from a Banach space $X$ into $X$. Then the following statements hold.

(i) For every positive integer $n$, the $n$-th power $S^{n}$ of $S$ is also a Noether operator such that $\kappa\left(S^{n}\right)=n \kappa(S)$.

(ii) For every strictly singular operator $A: X \rightarrow X$, the operator $S+A$ is a Noether operator such that $\kappa(S+A)=\kappa(S)$.

Let $1<p<\infty$. Let $\lambda$ denote Lebesgue measure in the open interval $\Omega=$ ]$-1,1\left[\right.$. By $\mathcal{L}^{p}(\Omega)$ we denote the usual Banach space of functions $f$ on $\Omega$ (strictly speaking, equivalence classes of functions modulo $\lambda$-null functions) such that $f|f|^{p-1}$ is $\lambda$-integrable. The finite Hilbert transform $T_{p}: \mathcal{L}^{p}(\Omega) \rightarrow \mathcal{L}^{p}(\Omega)$ is defined by the Cauchy principal value

$$
\left(T_{p} f\right)(t)=\lim _{\varepsilon \downarrow 0}\left[\int_{-1}^{t-\varepsilon}+\int_{t+\varepsilon}^{1}\right] \frac{f(\tau)}{\pi(\tau-t)} d \lambda(\tau), \quad t \in \Omega
$$

for every $f \in \mathcal{L}^{p}(\Omega)$. Then $T_{p}$ is a continuous linear operator by the M. Riesz theorem; the details can be found in [2, Section 13], for example.

LEMMA 2 .

(i) If $1<p<2$, then $T_{p}$ is a Noether operator such that $\kappa\left(T_{p}\right)=1$.

(ii) The operator $T_{2}$ is not a Noether operator; its range $\mathcal{R}\left(T_{2}\right)$ is a proper dense subspace of $\mathcal{L}^{2}(\Omega)$.

(iii) If $2<p<\infty$, then $T_{p}$ is a Noether operator such that $\kappa\left(T_{p}\right)=-1$.

Proof: Statements (i) and (iii) are due to Söhngen [7]. See also [2, Section 13] and [5, Propositions 2.4 and 2.6] for alternative proofs.

The fact that $T_{2}$ is not a Noether operator has been proved in the general setting; see, for example, [1, Theorem IX.5.3] or [4, Theorem IV.5.1]. Alternatively that fact 
can easily be derived from the observation that $\mathcal{R}\left(T_{2}\right)$ does not contain the constant function 1. For a characterisation of $\mathcal{R}\left(T_{2}\right)$, see [5, Theorem 3.2].

For every $p \in] 1, \infty\left[\right.$, the identity operator $I_{p}: \mathcal{L}^{p}(\Omega) \rightarrow \mathcal{L}^{p}(\Omega)$ is clearly a Noether operator such that

$$
\kappa\left(I_{p}\right)=0 .
$$

We now present the main result.

THEOREM 3. Let $1<p<\infty$. Then the linear operator $T_{p}^{n}+I_{p}: \mathcal{L}^{p}(\Omega) \rightarrow \mathcal{L}^{p}(\Omega)$ is not strictly singular, especially it is not compact, for any positive integer $n$.

Proof: Fix a positive integer $n$ and let $A_{p}=T_{p}^{n}+I_{p}$.

Firstly assume that $1<p<2$. Then, by Lemmas 1 and 2 , the operator $T_{p}^{n}$ is a Noether operator such that

$$
\kappa\left(T_{p}^{n}\right)=n
$$

If $A_{p}$ were strictly singular, then by Lemma 1(ii), the Noether operator $T_{p}^{n}=\left(-I_{p}\right)+A_{p}$ would have index 0 because of (1). This contradicts (2), so that $A_{p}$ is not strictly singular.

Secondly, if $A_{2}$ were strictly singular, then $T_{2}^{n}=\left(-I_{2}\right)+A_{2}$ would be a Noether operator. However, this is not the case because the range of $T_{2}^{n}$ is a proper dense subspace of $\mathcal{L}^{2}(\Omega)$ by Lemma $2($ ii).

In the case when $2<p<\infty$, the operator $T_{p}^{n}$ is a Noether operator whose index is $-n$ by Lemmas 1 and 2 . So $A_{p}$ is not strictly singular because of (1) as in the first case.

\section{REFERENCES}

[1] I. Gohberg and N. Krupnik, Einführung in die Theorie des eindimensional singulären Integraloperatoren, (German translation) (Birkhäuser Verlag, Basel, Boston, Stuttgart, 1979).

[2] K. Jörgens, Linear integral operators, (English translation) (Pitman, Boston, London, Melbourne, 1982).

[3] J. Lindenstrauss and L. Tzafriri, Classical Banach spaces I (Springer-Verlag, Berlin, Heidelberg, New York, 1977).

[4] S.G. Mikhlin and S. Prössdorf, Singular integral operators, (English translation) (SpringerVerlag, Berlin, Heidelberg, New York, Tokyo, 1986).

[5] S. Okada and D. Elliott, 'The finite Hilbert transform in $L^{2}$ ', Math. Nachr. 153 (1991), 43-56.

[6] M. Riesz, 'Sur les fonctions conjuguées', Math. $Z$. 27 (1927), 218-244. 
[7] H. Söhngen, 'Zur Theorie der endlichen Hilbert-Transformation', Math. Z. 60 (1954), 31-51.

Department of Mathematics

University of Tasmania

GPO Box 252C

Hobart Tas 7001 